\section{Anti-inflammatory cardiovascular therapies take another hit}

A placebo-controlled trial of methotrexate in more than 4,700 atherosclerosis patients did not reduce the incidence of cardiovascular events, dealing another blow to hopes that anti-inflammatory therapies can control heart disease.

Methotrexate is an anti-inflammatory that is used for the treatment of rheumatoid arthritis and other indications. The drug did not reduce the incidence of nonfatal myocardial infarction, nonfatal stroke or cardiovascular death compared with placebo, show data from the failed CIRT trial, presented at the American Heart Association Scientific Sessions in November and published in the New England Journal of Medicine.

The negative results are a setback for a community that was re-invigorated in 2017 by the CANTOS trial of Novartis's anti-IL-1 $\beta$ therapy canakinumab. The anti-inflammatory antibody reduced the incidence of atherosclerotic events by $15 \%$, showed the 10,000-patient trial. The benefit was greatest in patients who showed the largest reductions in IL- 6 and CRP with treatment, buoying hopes that drugs that act on the IL-1-IL-6-CRP pathway could improve cardiovascular outcomes. Methotrexate is also thought to act on this pathway, prompting researchers to hope that the CIRT trial would validate the CANTOS findings. Instead, the CIRT investigators report that methotrexate did not reduce levels of IL-1, IL-6 or CRP.

CIRT investigators called these findings neutral, but informative. "The observations in CANTOS, CIRT and other trials highlight the importance of considering the mechanistic diversity of inflammatory pathways in atherosclerosis and of exploring approaches to their inhibition. Understanding these differences is likely to be crucial for future studies targeting inflammation in atherosclerosis," they write in the New England Journal of Medicine.

Other anti-inflammatory agents that have failed to improve cardiovascular outcomes include GlaxoSmithKline's now-discontinued lipoprotein-associated phospholipase A2 (Lp-PLA2) inhibitor darapladib. A large trial of colchicine, a nonspecific inhibitor of the NLRP3 inflammasome, is ongoing.

Separately, Novartis disclosed in October that the FDA has rejected a bid for a supplemental approval for canakinumab for cardiovascular risk reduction. The company is evaluating the regulatory complete response letter to decide on next steps for this drug.

Asher Mullard

\section{FDA approves first new flu drug in 20 years}

The FDA approved Roche and Shionogi's baloxavir marboxil for the treatment of acute uncomplicated influenza. The polymerase acidic endonuclease inhibitor is the first new flu drug to be approved since the 1999 approval of Roche's neuraminidase inhibitor oseltamivir.

The recent approval was based on two pivotal trials in which the drug bested placebo. In a first trial in 400 influenza subjects, the flu symptoms of baloxavir-treated patients lasted 50 hours, down from 78 hours in the placebo arm. In a larger 1,400 patient trial, the time to alleviation of symptoms was 54 hours with baloxavir compared with 80 hours for placebo. In 500 patients who received oseltamivir in this trial, the median time to alleviation of symptoms was also 54 hours, in line with the effects of baloxavir.
Whereas oseltamivir is administered twice daily for 5 days, baloxavir is administered as a single dose.

In an editorial in the New England Lournal of Medicine, the Centers for Disease Control and Prevention's Timothy Uyeki noted that the virological effects of baloxavir are cause for both optimism and concern. On the upside, clinical data suggest that the drug seems to reduce the viral replication of influenza better than oseltamivir and placebo, suggesting that it has the "potential for reducing influenza virus spread". However, treatment with the new drug also led to the emergence of viral escape mutants with reduced drug susceptibility.

These results "should be viewed as a first step and the findings tempered by the need for data on baloxavir efficacy and safety," wrote Uyeki. Further studies are needed to look at the efficacy of baloxavir and oseltamivir in combination, he wrote, and to see whether the new drug reduces viral transmission when used in practice.

Asher Mullard

\section{RNAi deal flow heats up}

A recent flurry of deals highlighted growing industry interest in RNAi-based drugs that can silence disease-causing genes, just months after the FDA approved Alnylam's first-in-modality RNAi drug patisiran for hereditary transthyretin-mediated amyloidosis.

In October, Johnson \& Johnson partnered with Arrowhead Pharmaceuticals for rights to develop the phase I/II candidate ARO-HBV for the treatment of chronic hepatitis $B$ viral infection. The deal included an upfront payment of US $\$ 175$ million to Arrowhead, a $\$ 75$ million equity investment in the biotech and up to $\$ 3.5$ billion in potential milestone payments.

Later in the month, Alexion partnered with Dicerna Pharmaceuticals for rights to collaborate on the discovery and development of RNAi-based therapies for complement-mediated diseases. This included rights to two preclinical RNAi molecules and exclusive options to two additional targets in the complement pathway. Alexion made an upfront payment of $\$ 22$ million, a $\$ 15$ million equity investment in Dicerna and committed to approval-related milestone payments of up to $\$ 105$ million per target.

Eli Lilly is also getting into the RNAi space, partnering with Dicerna to collaborate on cardiometabolic, neurodegeneration and pain targets. Eli Lilly made an upfront payment of $\$ 100$ million and an equity investment of $\$ 100$ million to collaborate on more than ten targets. Dicerna will also be eligible for up to $\$ 350$ million in milestones per target.

The enthusiasm for RNAi-based drugs is driven by hopes that these gene-silencing oligonucleotides can open up targets that have eluded other drug modalities. At least six RNAi-based products are currently in phase III development, and next-generation candidates stand to offer better access to targets beyond the liver, better therapeutic indices and better delivery options.

Asher Mullard 